University of Wollongong

Research Online

Faculty of Engineering - Papers (Archive)

Faculty of Engineering and Information

Sciences

$1-1-2011$

\title{
Bulk and surface field-induced optical rectification from (11N) zincblende crystals in a quasireflection geometry
}

Krunal Radhanpura

University of Wollongong, krunal@uow.edu.au

Stuart Hargreaves

University of Wollongong, sh64@uow.edu.au

Roger A. Lewis

University of Wollongong, roger@uow.edu.au

Follow this and additional works at: https://ro.uow.edu.au/engpapers

Part of the Engineering Commons

https://ro.uow.edu.au/engpapers/561

\section{Recommended Citation}

Radhanpura, Krunal; Hargreaves, Stuart; and Lewis, Roger A.: Bulk and surface field-induced optical rectification from $(11 \mathrm{~N})$ zincblende crystals in a quasireflection geometry 2011.

https://ro.uow.edu.au/engpapers/561

Research Online is the open access institutional repository for the University of Wollongong. For further information contact the UOW Library: research-pubs@uow.edu.au 


\title{
Bulk and surface field-induced optical rectification from $(11 N)$ zincblende crystals in a quasireflection geometry
}

\author{
K. Radhanpura, S. Hargreaves, ${ }^{*}$ and R. A. Lewis \\ Institute for Superconducting and Electronic Materials, University of Wollongong, Wollongong, New South Wales 2522, Australia
}

(Received 29 October 2010; published 31 March 2011)

\begin{abstract}
In a previous article, we presented a generalized expression for second-order bulk and third-order surfacefield-induced optical rectification for zincblende $\overline{4} 3 \mathrm{~m}$ crystal faces with arbitrary Miller indices $(h k l)$ along with experimental data for $(11 N) A$ and $(11 N) B$ GaAs in transmission geometry. We now expand the results to quasireflection geometry, with angles of incidence and detection of $45^{\circ}$. While this geometry introduces a $p$-polarized signal component due to mechanisms other than optical rectification, such as photocarrier acceleration by the surface depletion field, the azimuthal angle dependence of the optical rectification component yields further insight into the crystallographic orientation and surface properties of the sample.
\end{abstract}

DOI: 10.1103/PhysRevB.83.125322

PACS number(s): 78.20.Jq, 78.68.+m, 42.70.Nq

\section{INTRODUCTION}

The pulsed generation of terahertz radiation by samples under excitation from ultra-short near-infrared pulses has facilitated the development of time-domain spectroscopy and imaging in the previously elusive terahertz region of the electromagnetic spectrum, considered here to range from 0.3 to $3 \mathrm{THz}$. Emission is known to depend strongly on the experimental geometry used, such as the orientation of the excitation beam and detector relative to the sample. Additionally, effects such as electric field screening ${ }^{1}$ may need to be considered. Emission may be due to one or more of several mechanisms, each of which may have a distinct dependence on the experimental geometry employed.

For instance, the application of an external electric bias to the semiconductor surface with a photolithographically grown photoconductive switch, ${ }^{2,3}$ or with simple silver paint contacts, ${ }^{4,5}$ results in a terahertz electric field in the surface plane, parallel to the bias field, as above-bandgap optical pulses generate free carriers which are in turn accelerated by the bias field. Photoconductive emitters are most commonly employed in a straight-through transmission geometry.

Emission may also occur due to the transport of photogenerated free-carriers at the semiconductor surface ${ }^{6}$ in the absence of an external biasing field. Such emission may be due to the current surge effect, where photocarriers are accelerated by an intrinsic surface depletion field which arises due to band bending by Fermi-level pinning of the surface states, ${ }^{7}$ which is typically observed for wide-bandgap materials such as $\mathrm{GaAs}^{8}$ and $\mathrm{InP}^{9,10}$ It may also be due to differing diffusion rates between photogenerated electrons and holes at the surface, known as the photo-Dember effect and typically observed in narrow-bandgap semiconductors with a high electron mobility such as InAs, ${ }^{11,12} \mathrm{InSb},{ }^{13}$ and GaSb. ${ }^{14}$ While the effects are often mutually exclusive, ${ }^{15}$ it has been shown that either mechanism can dominate in GaAs under the appropriate experimental conditions. ${ }^{8}$ For both the current surge and photo-Dember effects, the transient terahertz electric field is normal to the semiconductor surface and thus will not contribute to emission in a transmission geometry. A quasireflection geometry is instead employed, allowing for the detection of the $p$-polarized signal.
Finally, the nonlinear processes of bulk ${ }^{16}$ and surfacefield-induced ${ }^{17,18}$ optical rectification are known to depend strongly on not only the angles of incidence and detection but additionally on the polarization of the excitation beam, the crystallographic plane on which the surface is cut, and the azimuthal angle of the sample about its surface normal. The azimuthal angle dependence of the emitted terahertz signal is an identifying characteristic of optical rectification, and our previous article (Hargreaves, Radhanpura, and Lewis, 2009, ${ }^{19}$ henceforth referred to as HRL) presented a general expression for the azimuthal angle dependence of both bulk and surface optical rectification in a zincblende crystal of arbitrary $(h k l)$ crystallographic surface plane. In presenting the theory, we compared the calculated expression to experimental data for $(11 N) \mathrm{GaAs}$ in a straight-through transmission geometry and have since demonstrated a significant enhancement of the surface-field-induced signal in a (113) $B \mathrm{GaBi}_{0.035} \mathrm{As}_{0.965}$ epilayer relative to the (113) $B$ GaAs substrate. ${ }^{20}$

In this article, we extend the previous work to a quasireflection geometry, including an experimental analysis of $(11 N)$ $\mathrm{GaAs}$ and a discussion of the results in the context of the recently developed theory for $(11 N)$ zincblende samples. To set the scene, Fig. 1 presents results for (115)A GaAs in both transmission and reflection, with a diagram of each geometry included as insets. We include data for (110), (111), and the high-index (112), (113), (114), and (115) faces of GaAs. Additionally, we include data for opposing $A$ and $B$ faces.

\section{THEORY}

Bulk optical rectification in the crystallographic frame may be calculated using the second-order susceptibility tensor $\chi_{i j k}^{(2)}(\Omega ;-\omega, \omega)$, where $\Omega$ is the terahertz difference frequency and $\omega$ is the excitation frequency. In materials with $\overline{4} 3 m$ zincblende crystal symmetry, the tensor contains only a single nonvanishing term $d_{14}=\chi_{i j k}^{(2)}[i \neq j \neq k]$, such that the bulk terahertz polarization is given by

$$
\left[\begin{array}{c}
P_{x}^{\text {bulk }} \\
P_{y}^{\text {bulk }} \\
P_{z}^{\text {bulk }}
\end{array}\right]=2 d_{14}\left[\begin{array}{cc}
E_{y} & E_{z} \\
E_{x} & E_{z} \\
E_{x} & E_{y}
\end{array}\right],
$$


where $P_{i}$ and $E_{i}$ are components of the terahertz polarization and the excitation electric field, respectively. Similarly, the surface-field-induced effect may be calculated using the third- order susceptibility tensor $\chi_{i j k z}^{(3)}(\Omega ;-\omega, \omega, 0)$, with the zero term corresponding to the DC surface depletion field. The surface-field-induced terahertz polarization is given by ${ }^{18}$

$$
\left[\begin{array}{c}
P_{x}^{\text {surf }} \\
P_{y}^{\text {surf }} \\
P_{z}^{\text {surf }}
\end{array}\right]=\left[\begin{array}{l}
\alpha E_{0}^{2} F_{x}+\beta\left(E_{x} F_{x}+E_{y} F_{y}+E_{z} F_{z}\right) E_{x}+\gamma E_{x}^{2} F_{x} \\
\alpha E_{0}^{2} F_{y}+\beta\left(E_{x} F_{x}+E_{y} F_{y}+E_{z} F_{z}\right) E_{y}+\gamma E_{y}^{2} F_{y} \\
\alpha E_{0}^{2} F_{z}+\beta\left(E_{x} F_{x}+E_{y} F_{y}+E_{z} F_{z}\right) E_{z}+\gamma E_{z}^{2} F_{z}
\end{array}\right],
$$

where $\alpha=3 \chi_{z x x z}, \beta=6 \chi_{z z x x}$, and $\gamma=3\left(\chi_{z z z z}-\chi_{z x x z}-\right.$ $\left.2 \chi_{z z x x}\right)$ and $F_{i}$ describes components of the surface depletion field. By transforming the excitation electric field and the surface depletion field into the crystallographic frame of reference, calculating the terahertz polarization due to optical rectification, and transforming the polarization back into the laboratory frame, HRL calculated the azimuthal angle dependence of the total induced polarization due to second-order bulk and third-order surface-field-induced components. The total response was found to consist of sinusoidal components up to $3 \theta$ as follows:

$$
\begin{gathered}
{\left[\begin{array}{c}
P_{x^{\prime \prime}}^{\text {bulk }} \\
P_{y^{\prime \prime}}^{\text {bulk }} \\
P_{z^{\prime \prime}}^{\text {bulk }}
\end{array}\right]=\left[\begin{array}{l}
G_{11}+G_{12} \cos \theta+G_{13} \sin \theta+G_{14} \cos 2 \theta+G_{15} \sin 2 \theta+G_{16} \cos 3 \theta+G_{17} \sin 3 \theta \\
G_{21}+G_{22} \cos \theta+G_{23} \sin \theta+G_{24} \cos 2 \theta+G_{25} \sin 2 \theta+G_{26} \cos 3 \theta+G_{27} \sin 3 \theta \\
G_{31}+G_{32} \cos \theta+G_{33} \sin \theta+G_{34} \cos 2 \theta+G_{35} \sin 2 \theta+G_{36} \cos 3 \theta+G_{37} \sin 3 \theta
\end{array}\right],} \\
{\left[\begin{array}{c}
P_{x^{\prime \prime}}^{\text {surf }} \\
P_{y^{\prime \prime}}^{\text {surf }} \\
P_{z^{\prime \prime}}^{\text {surf }}
\end{array}\right]=\left[\begin{array}{l}
V_{11}+V_{12} \cos \theta+V_{13} \sin \theta+V_{14} \cos 2 \theta+V_{15} \sin 2 \theta+V_{16} \cos 3 \theta+V_{17} \sin 3 \theta \\
V_{21}+V_{22} \cos \theta+V_{23} \sin \theta+V_{24} \cos 2 \theta+V_{25} \sin 2 \theta+V_{26} \cos 3 \theta+V_{27} \sin 3 \theta \\
V_{31}+V_{32} \cos \theta+V_{33} \sin \theta+V_{34} \cos 2 \theta+V_{35} \sin 2 \theta+V_{36} \cos 3 \theta+V_{37} \sin 3 \theta
\end{array}\right]}
\end{gathered}
$$

The coefficients $G_{i j}$ and $V_{i j}$ depend on the Miller indices of the crystallographic plane $(h, k$, and $l)$, the refracted angle of incidence $\phi_{\mathrm{NIR}}$, the polarization angle relative to the plane of incidence $\Phi$, and the pump electric field magnitude $E_{0}$. Furthermore, the bulk optical rectification depends on the second-order susceptibility coefficient $d_{14}$, and the surface-field-induced signal depends on the surface field $F_{0}$ and the third-order susceptibility coefficient $\gamma$. The values of these coefficients may be found in Appendix A of HRL. The polarization components $P_{x^{\prime \prime}}, P_{y^{\prime \prime}}$, and $P_{z^{\prime \prime}}$ are those in the laboratory-fixed frame of reference, with the detected signal corresponding to a rotation of the double-primed components by the refracted angle of detection $\phi_{\mathrm{THz}}$ into the quadruple-primed frame $\left(x^{\prime \prime \prime \prime}, y^{\prime \prime \prime \prime}, z^{\prime \prime \prime \prime}\right)$. The $\alpha$ and $\beta$ surface-field-induced components are azimuthally invariant and are estimated to be small compared to the current surge effect and have been neglected.

The coordinates were chosen such that $\mathbf{x}^{\prime \prime \prime}$ lies along the surface normal or along the $(h k l)$ crystallographic direction for the $A$ face and $(\bar{h} \bar{k} \bar{l})$ for the opposing $B$ face. The distinction between opposing $A$ and $B$ faces is outlined in Fig. 2. Also, $\hat{\mathbf{y}}^{\prime \prime \prime}$ lies along the $[\bar{k} h 0]$ and $[k \bar{h} 0]$ directions for the $A$ and $B$ faces, respectively, constraining $\mathbf{z}^{\prime \prime \prime}$ to lie along the $\left[-h l,-k l, h^{2}+k^{2}\right]$ direction for both faces. The laboratory-fixed double-primed frame is chosen such that $\hat{\mathbf{x}^{\prime \prime}}$ also lies along the surface normal, and $\hat{\mathbf{y}}^{\prime \prime}$ lies along the projection of the excitation beam onto the surface. The angle $\theta$ corresponds to a clockwise azimuthal rotation of the sample about the surface normal, with $\theta=0$ corresponding to coincident double-primed and triple-primed frames. Figure 3 illustrates the crystal-fixed triple-primed frame for opposing $A$ and $B$ faces.

Figure 4 illustrates the theoretical distinction between opposing $A$ and $B$ faces using the example of the (112) face. A reversal of the dominant $\theta$ and $3 \theta$ terms between faces is evident for the surface-field induced effect but not for the bulk effect. On the other hand, the $2 \theta$ term and the signal offset reverse between faces for the bulk effect but not for the surface effect. Hence the choice of face is expected to have a pronounced effect on the combined optical rectification signal. This is not observed for (110) cuts due to the equivalence of opposing faces, and with the threefold rotational symmetry of the (111) face, the choice of $A$ or $B$ faces corresponds only to a change in the amplitude of the azimuthal angle dependence and not to its shape. Hence high-index samples yield insights into the surface properties that are not available for low-index samples.

\section{EXPERIMENT}

In contrast to the simple case of emitter-transmission geometry employed in HRL, a quasireflection geometry, with angles of incidence and detection of $45^{\circ}$, was employed. In principle, both optical rectification and current surge effects 


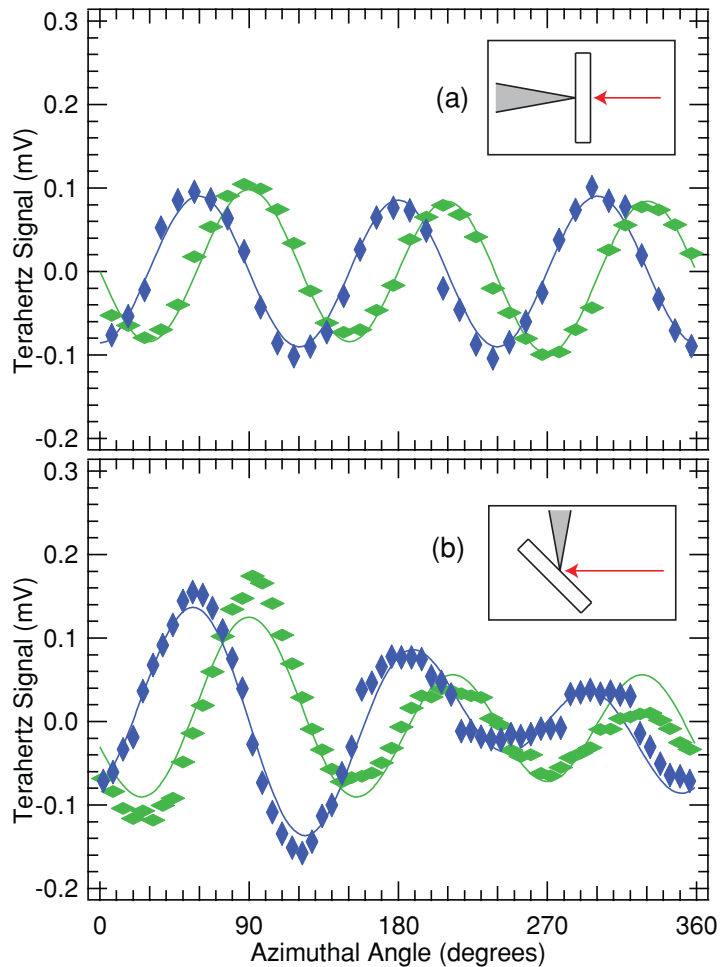

FIG. 1. (Color online) Results for the (115) $A$ face in (a) transmission and (b) quasireflection geometry. Green horizontal diamonds (color online) represent the $p$-polarized or horizontally polarized signal, blue vertical diamonds represent the $s$-polarized or vertically polarized signal.

may be observed in this geometry. Several GaAs samples cut along $(11 N)$ planes were tested using $p$-polarized sub12 -fs excitation pulses at a center wavelength of $790 \mathrm{~nm}$ $(h v=1.57 \mathrm{eV})$, detected electro-optically with a $1 \mathrm{~mm}$ (110) ZnTe wafer in a conventional time-domain spectroscopy



FIG. 2. (Color online) The III-V zincblende crystal structure, as seen from the [1필 direction, illustrating the $A$ and $B$ surfaces of several $(11 N)$ planes. Black atoms correspond to group III atoms, white atoms correspond to group $\mathrm{V}$ atoms. Note that due to the symmetry of (001) and (110) planes (and their equivalents), both sides of cuts along these planes are equivalent and there is no need to specify "A" or "B" faces. The [001] and [110] directions are included for reference.

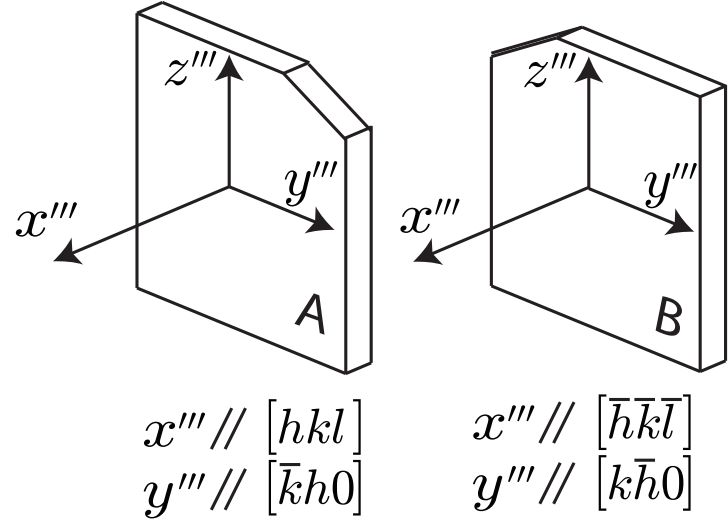

FIG. 3. Opposing $A$ and $B$ faces of a semiconductor crystal. Included are the crystal-fixed triple-primed coordinates.

arrangement. The signal is measured as the differential voltage output of a balanced photodiode pair, which is directly proportional to the $\mathrm{THz}$ field in the ZnTe crystal.

The $p$-polarized pump beam corresponds to $\Phi=0$, and by using the refractive index of GaAs at $790 \mathrm{~nm}(n=3.67),{ }^{21}$ one finds that $\phi_{\text {NIR }}=11.1^{\circ}$. Similarly, using the low-frequency refractive index of $n=3.58,{ }^{21}$ the refracted angle of detection $\phi_{\mathrm{THz}}$ equals $11.4^{\circ}$. Separate measurements were taken on both $A$ and $B$ faces for each $(11 N)$ sample $(N=0$ to $N=5)$.

For each azimuthal angle, separate time-domain scans over the main terahertz peak were taken, with the terahertz signal amplitude taken to be the peak-to-peak value of the differential



FIG. 4. (Color online) Calculated terahertz signal amplitude in (112) GaAs as a function of azimuthal angle due to (a) bulk optical rectification and (b) surface-field-induced optical rectification. A distinction between the $A$ face (solid lines) and $B$ face (dashed lines) is evident for the (112) face; this is not expected for the (110) face. 
voltage signal detected by the photodiode pair. The process was repeated for alternate terahertz polarizations ( $s$ and $p$ isolated with a wire-grid polarizer) and for each GaAs sample. While the azimuthal orientation of the samples was not known beforehand, it could be inferred from the orientation of best fit between theoretical and experimental data, allowing us to identify the crystallographic directions $(\bar{k} h 0)$ and $(k \bar{h} 0)$ in the surface plane.

If the absorption length is sufficiently short relative to the depletion width, the surface depletion field can be considered constant over the absorption length, and $\gamma F_{0} / d_{14}$ may be determined experimentally by comparing the bulk and surface contributions to the signal. The absorption length for GaAs at $790 \mathrm{~nm}$ is ${ }^{22} \alpha^{-1}=7.0 \times 10^{-7} \mathrm{~m}$, and according to the Schottky model, ${ }^{23}$ the depletion width is given by $W=\left(2 \epsilon_{0} \epsilon_{r} V_{\mathrm{bi}} / q N\right)^{1 / 2}$, where $\epsilon_{0}$ and $\epsilon_{r}$ correspond to the permittivity of free space and the static relative permittivity respectively, $V_{\mathrm{bi}}$ is the built-in potential, $q$ is the carrier charge, and $N$ is the carrier density. With a built-in potential of $0.75 \mathrm{~V}$ for SI GaAs, ${ }^{24}$ we expect the absorption depth to be less than the depletion width if $N<1.9 \times 10^{15} \mathrm{~cm}^{-3}$, which is the case for the samples measured.

\section{RESULTS AND DISCUSSION}

Waterfall plots of the $s$-polarized and $p$-polarized timedomain signals for (112) $A$ GaAs are given as Figs. 5 and 6 , respectively. It is clear that the $s$-polarized signal and the $p$-polarized signal are distinct. The $p$-polarized signal is stronger, with optical rectification contributing to a proportionally small angular dependence relative to the azimuthally invariant term due to photocarrier acceleration. The polarity of the the $p$-polarized signal is consistent with both the photoDember effect and current surge from a surface depletion field pointing out of the surface plane; however, under our experimental conditions, it is believed to be due primarily to the current surge effect. It is also apparent that the timedomain profile of the $s$-polarized signal shows greater temporal symmetry than the $p$-polarized signal.



FIG. 5. (Color online) A waterfall plot of the $s$-polarized timedomain electric field profile of $\mathrm{THz}$ emission from (112)A GaAs, illustrating the variation as the azimuthal angle of the sample is varied.



FIG. 6. (Color online) A waterfall plot of the $p$-polarized timedomain electric field profile of THz emission from (112) $A$ GaAs as the azimuthal angle is varied. Since the strong current surge component does not depend on the azimuthal angle, the azimuthal variation is less pronounced.

We turn our attention to the azimuthal angle dependence of the (110) "A" and "B" faces in Fig. 7. While the bulk structure of the two faces is equivalent due to crystal symmetry, both faces are included for completeness. Both faces have a significant $p$-polarized offset due to the azimuthally invariant current surge effect; however, the offset was slightly stronger for the nominal " $\mathrm{A}$ " face with an offset of $1.61 \pm 0.02 \mathrm{mV}$ compared to $1.21 \pm 0.02 \mathrm{mV}$ for the " $\mathrm{B}$ " face. Although there was significant noise in the $p$-polarized (110)" $\mathrm{B}$ " response, the $s$-polarized response for both faces and the $p$-polarized (110)

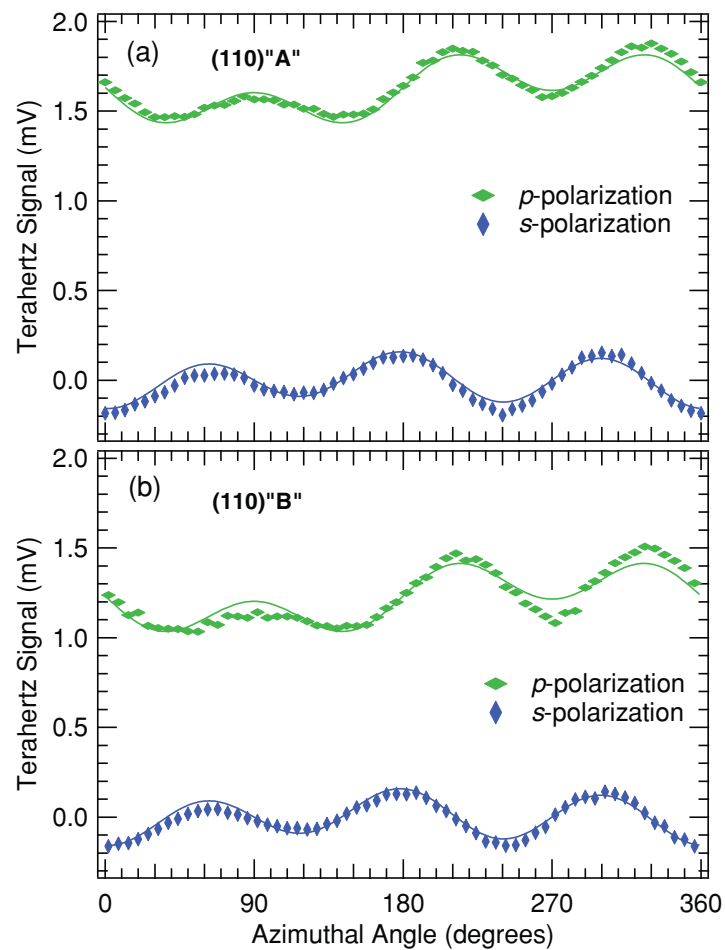

FIG. 7. (Color online) Measured terahertz signal amplitude in opposing (a) "A" and (b) "B" GaAs (110) faces as a function of the azimuthal angle of the sample. Due to the symmetry of the (110) plane, the two faces are theoretically equivalent. 


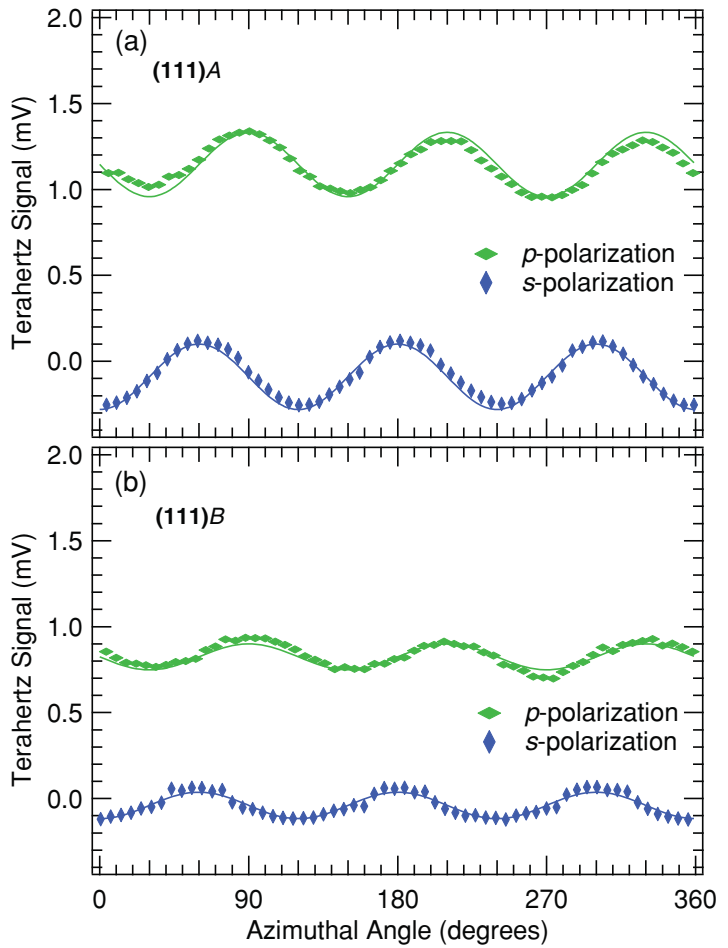

FIG. 8. (Color online) Measured terahertz signal amplitude in (a) (111) $A$ and (b) (111) $B$ GaAs as a function of the azimuthal angle of the sample.

"A" response proved a good fit to theory with fitting parameters of $Z=(170 \pm 30) \mathrm{mV}$ [where $Z$ is the ratio between the detected signal and the dimensionless polarization expression $\left.P /\left(d_{14} E_{0}^{2}\right)\right]$ and $\gamma F_{0} / d_{14}=1.5 \pm 0.8$ respectively.

By retaining these fitting parameters for (111) samples, a close fit between theoretical and experimental results is again observed, as given in Fig. 8. Due to the threefold rotational symmetry of the (111) face, the azimuthal angle dependence contains only $\sin 3 \theta$ and $\cos 3 \theta$ terms. For the $A$ face, the surface component is in phase with the bulk term, leading to an enhancement of the total signal. For the $B$ face, the two components are out of phase and the total signal is reduced. The current surge offset is slightly less than for the (110) faces, at $1.15 \pm 0.02$ and $0.79 \pm 0.02 \mathrm{mV}$ for the $A$ and $B$ faces, respectively.

Results for the high-index (112), (113), (114), and (115) samples are presented in Figs. 9 and 10 for $s$ and $p$ polarizations, respectively, still retaining the previous fitting parameters. For the $s$-polarized signal, the $A$ face gave stronger results than the $B$ face as predicted by theory; however, due to an increased signal-to-noise ratio for the $B$ face, the $A$ face had a closer correspondence between theoretical and experimental data.

For the $p$-polarized signal, the predicted distinction between the $A$ and $B$ faces in the shape of the azimuthal angle dependence is observed. While the current surge offsets for the (112) samples were comparable to the (110) samples, at $1.37 \pm 0.02$ and $1.67 \pm 0.02 \mathrm{mV}$ for the $A$ and $B$ faces respectively, the offsets for both faces of the higher-index (113), (114), and (115) samples were all found to lie in the range of 0.57 to $0.68 \mathrm{mV}$.

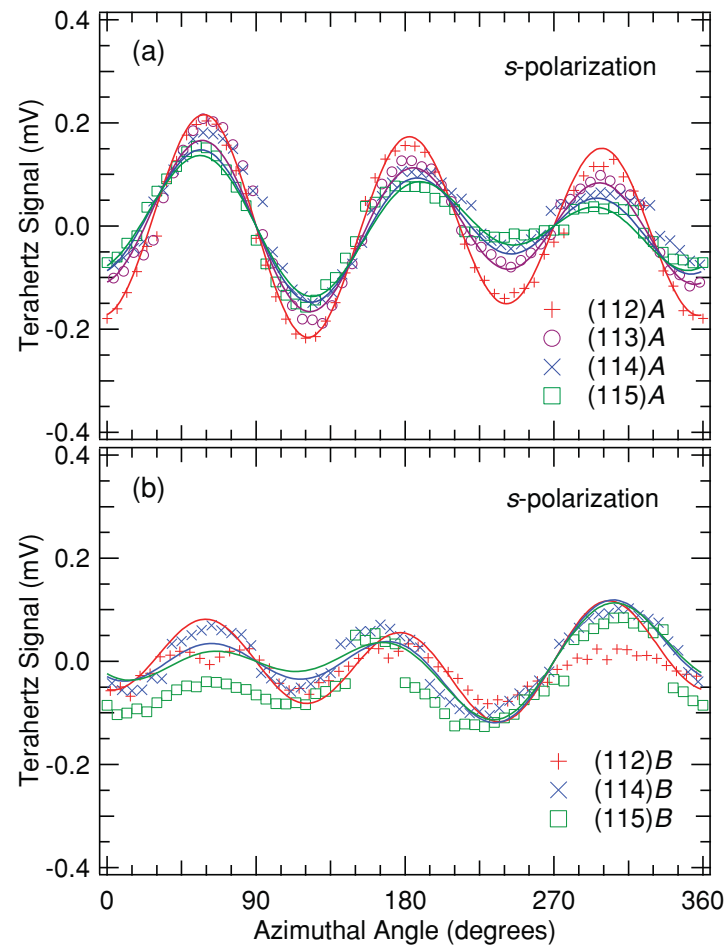

FIG. 9. (Color online) Measured $s$-polarized terahertz signal amplitude in (112), (113), (114), and (115) GaAs as a function of azimuthal angle. Both (a) $A$ and (b) $B$ faces are included.

Both the surface-field-induced optical rectification and current surge components of the results indicate a surface field pointing outward from the surface, consistent with the

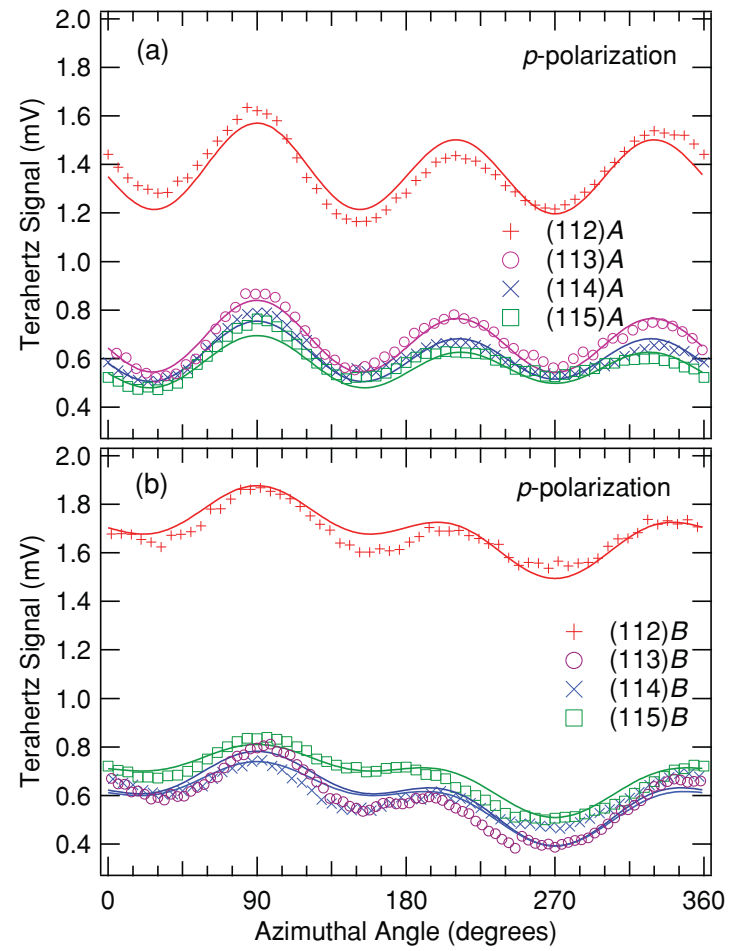

FIG. 10. (Color online) Measured p-polarized terahertz signal amplitude in (112), (113), (114), and (115) GaAs. Both (a) $A$ and (b) $B$ faces are included. 
TABLE I. The $p$-polarized signal offset for each sample, believed to be due primarily to the current surge effect. Values correspond to the peak-to-peak signal from the photodiode detector pair and are proportional to the peak-to-peak $\mathrm{THz}$ electric field at the $\mathrm{ZnTe}$ detector crystal.

\begin{tabular}{lcc}
\hline \hline Surface plane & $A$ offset $(\mathrm{mV})$ & $B$ offset $(\mathrm{mV})$ \\
\hline$(110)$ & $1.61 \pm 0.02$ & $1.21 \pm 0.02$ \\
$(111)$ & $1.15 \pm 0.02$ & $0.79 \pm 0.02$ \\
$(112)$ & $1.37 \pm 0.02$ & $1.67 \pm 0.02$ \\
$(113)$ & $0.68 \pm 0.02$ & $0.57 \pm 0.02$ \\
$(114)$ & $0.62 \pm 0.02$ & $0.60 \pm 0.02$ \\
$(115)$ & $0.59 \pm 0.02$ & $0.67 \pm 0.02$ \\
\hline \hline
\end{tabular}

surface depletion field for our $n$-type samples. There was an apparent variation in the current surge component between the samples, as demonstrated in Table I. This is likely due to minor variations in the surface properties such as the carrier concentration. Since both the surface-field-induced optical rectification component and the current surge component depend directly on the surface field intensity, a correlation between the two contributions is expected; however, due to uncertainties in the surface-field-induced term, such a correlation could not be conclusively identified.

Part of the uncertainty in fitting the results to the theoretical predictions comes from the complication of the differing timedomain profiles of the current surge and optical rectification signal components (see Figs. 5 and 6), such that the total peak-to-peak signal may not correspond to the sum of the peak-to-peak signal for each component. Drift and noise in the near infrared laser was also evident. There may also be as-yet unidentified azimuthal angle-dependent contributions to the total signal, as observed previously for (100) InP. ${ }^{25}$

\section{CONCLUSIONS}

The azimuthal angle dependence of the time-domain electric field profile of emitted terahertz radiation from $\mathrm{GaAs}$ in a quasireflection geometry has been observed for several (11N)-cut samples, with $N$ ranging from 0 to 5 .

As well as a clear contribution to the $p$-polarized signal from the current surge effect, the characteristic azimuthal variation of optical rectification is also observed. We find that the results may be explained as a sum of current surge, bulk optical rectification, and surface optical rectification components, using our previously developed theory for optical rectification in high-Miller-index zincblende crystals.

We observe a clear distinction between crystallographic planes and between opposing $A$ and $B$ faces, measure the direction and relative intensity of the surface depletion field in each sample, and identify the in-plane crystallographic direction [110]. Optical rectification may hence be used in the analysis of semiconductor surface properties and to identify the precise crystallographic orientation of a sample.

\section{ACKNOWLEDGMENTS}

This work was supported by the Australian Research Council and by the University of Wollongong. We thank M. Henini for provision of the samples used in this work.

\footnotetext{
*sh64@uowmail.edu.au

${ }^{1}$ H. Heesel, S. Hunsche, H. Mikkelsen, T. Dekorsy, K. Leo, and H. Kurz, Phys. Rev. B 47, 16000 (1993).

${ }^{2}$ P. LeFur and D. H. Auston, Appl. Phys. Lett. 28, 21 (1976).

${ }^{3}$ C. Fattinger and D. Grischkowsky, Appl. Phys. Lett. 53, 1480 (1988).

${ }^{4}$ D. Schönherr, H. L. Hartnagel, S. Hargreaves, R. A. Lewis, and M. Henini, Semicond. Sci. Technol. 23, 105012 (2008).

${ }^{5}$ G. Zhao, R. N. Schouten, N. van der Valk, W. T. Wenckebach, and P. C. M. Planken, Phys. Med. Biol. 47, 3699 (2002).

${ }^{6}$ T. Dekorsy and T. Pfeifer, W. Kütt, H. Kurz, Phys. Rev. B 47, 3842 (1993).

${ }^{7}$ H. Takahashi, A. Quema, M. Goto, S. Ono, and N. Sarukura, Jap. J. Appl. Phys. 42, L1259 (2003).

${ }^{8}$ J. N. Heyman, N. Coates, A. Reinhardt, and G. Strasser, Appl. Phys. Lett. 83, 5476 (2003).

${ }^{9}$ X.-C. Zhang and D. H. Auston, J. Appl. Phys. 71, 326 (1992).

${ }^{10}$ M. Nakajima, Y. Oda, and T. Suemoto, Appl. Phys. Lett. 85, 2694 (2004).

${ }^{11}$ P. Gu, M. Tani, S. Kono, K. Sakai, and X.-C. Zhang, J. Appl. Phys. 91, 5533 (2002).

${ }^{12}$ R. Mendis, M. L. Smith, L. J. Bignell, R. E. M. Vickers, and R. A. Lewis, J. Appl. Phys. 98, 126104 (2005).
}

${ }^{13}$ S. Kono, P. Gu, M. Tani, and K. Sakai, Appl. Phys. B: Lasers Opt. 71, 901 (2000)

${ }^{14}$ R. Ascázubi, C. Shneider, I. Wilke, R. Pino, and P. S. Dutta, Phys. Rev. B 72, 045328 (2005).

${ }^{15}$ F. Nastos and J. E. Sipe, Phys. Rev. B 74, 035201 (2006).

${ }^{16}$ A. Rice, Y. Jin, X. F. Ma, X.-C. Zhang, D. Bliss, J. Larkin, and M. Alexander, Appl. Phys. Lett. 64, 1324 (1994).

${ }^{17}$ M. Reid, I. V. Cravetchi, and R. Fedosejevs, Phys. Rev. B 72, 035201 (2005).

${ }^{18}$ V. L. Malevich, A. Krotkus, A. Bičiūnas, and V. Pačebutas, J. Appl. Phys. 104, 113117 (2008).

${ }^{19}$ S. Hargreaves, K. Radhanpura, and R. A. Lewis, Phys. Rev. B 80, 195323 (2009).

${ }^{20}$ K. Radhanpura, S. Hargreaves, R. A. Lewis, and M. Henini, Appl. Phys. Lett. 94, 251115 (2009).

${ }^{21}$ J. S. Blakemore, J. Appl. Phys. 53, R123 (1982).

${ }^{22}$ E. D. Palik, Handbook of Optical Constants of Solids (Academic Press, New York, 1985).

${ }^{23}$ S. M. Sze, Physics of Semiconductor Devices, 2nd ed. (Wiley, New York, 1991).

${ }^{24}$ J. Qi, M. S. Yeganeh, I. Koltover, A. G. Yodh, and W. M. Theis, Phys. Rev. Lett. 71, 633 (1993).

${ }^{25}$ S. Hargreaves and R. A. Lewis, Appl. Phys. Lett. 93, 242101 (2008). 